\title{
QUANTIFICAÇÃO E ANÁLISE COMPARATIVA DO TEOR DE METANOL EM AMOSTRAS DE CACHAÇAS DE DIFERENTES ORIGENS
}

\section{QUANTIFICATION AND COMPARATIVE ANALYSIS OF THE CONTENT OF METHANOL IN ALCOHOLIC DRINK FROM DIFFERENT ORIGINS OF}

Amandio Oliveira da Silva Júnior, Angélica Augusta Grigoli, Daniel Ângelo Macena

Universidade do Oeste Paulista - UNOESTE, Bacharelado em Química, Presidente Prudente, SP.

E-mail: danielangelo@live.com.

RESUMO - O modo de fabricação da cachaça está diferenciado na escala, equipamentos disponíveis, e o controle do produto final. Durante o processo de obtenção da bebida, são formados outros tipos de álcoois sendo eles desejáveis ou não. Dentre os álcoois indesejáveis está o metanol, um álcool de baixo peso molecular, responsável por intoxicações agudas que pode causar cegueira e em alguns casos evoluem levando a óbito, através de inalação, absorção pela pele e/ou ingestão. $\mathrm{O}$ objetivo da pesquisa foi identificar e quantificar o metanol em cachaças brasileiras de diferentes origens e comparar os resultados com a legislação brasileira. O método utilizado foi cromatografia gasosa por headspace e os resultados foram negativos para todas as amostras. Concluiu-se que as cachaças analisadas estavam de acordo com a legislação brasileira devido à ausência de metanol.

Palavras-chave: Metanol; Intoxicação; Cromatografia Gasosa.

ABSTRACT - The cachaça manufacturing mode is different in scale, equipment available and the control of the final product. During the process for obtaining the beverage, are formed other alcohols they are desirable or not. Among the undesirable alcohols, it is methanol, a low molecular weight alcohol responsible for acute intoxication, which can cause blindness and in some cases can leading to death through inhalation, ingestion and skin absorption. The objective of the research was to identify and quantify the methanol in Brazilian cachaça from different sources and compare the results with Brazilian law. The method used was gas chromatography by headspace and the results were negative for all samples. We concluded that the analyzed

Recebido em: 23/08/2016 Revisado em: 27/08/2016 Aprovado em: 05/09/2016 cachaças were in accordance with Brazilian law in the absence of methanol.

Keywords: Methanol; Intoxication; Gas Chromatography. 


\section{INTRODUÇÃO}

O cauim, assim conhecida a cachaça na época, era produzida pelos Tupinambás a partir de mandioca, milho ou caju, onde esses alimentos eram mastigados por mulheres virgens ou mulheres afastadas da vida sexual e logo cuspidos em um recipiente dando início ao processo de fermentação. Era uma bebida bastante consumida tanto por homens quanto por mulheres, servida em toda a tribo nas festas canibais antes de servir as carnes dos inimigos (GAMBINI, 2000).

A primeira versão do início da destilação são relatos de que os portugueses tomavam resíduos do caldo de cana, o que provavelmente após fermentação levariam ao mesmo efeito prazeroso. A segunda versão surgiu depois que por ventura um dos escravos que trabalhava na moenda de um engenho, resolveu provar o tal caldo de aparência repugnante que servia para alimentar os gados (MAZARO, 2004).

Nos dias de hoje a cachaça é a segunda bebida alcoólica mais consumida no Brasil, encontra-se abaixo da cerveja somente (MAIA, 1994).

Cachaça é a denominação típica e exclusiva da aguardente de cana-de-açúcar produzida no Brasil, com graduação alcoólica de 38 a $48 \%$ v/v a $20^{\circ} \mathrm{C}$, obtida pela destilação do mosto fermentado do caldo de cana de açúcar com características sensoriais peculiares, podendo ser adicionada de açúcares até $6 \mathrm{~g} / \mathrm{L}$, expresso em sacarose (SILVA; PORTELA; ARAÚJO, 2007).

Algumas substâncias podem ser consideradas referências no processo de envelhecimento. Durante a maturação de bebidas destiladas normalmente ocorre uma diminuição do $\mathrm{pH}$ e das concentrações de álcool metílico e de álcool etílico, enquanto que devido ao aumento da acidez, ocorre a alteração da cor e das concentrações de acetato de etila, de aldeído acético, de acetona e dos compostos fenólicos (taninos) (MIRANDA et al., 2008).

Diversos fatores influenciam na qualidade química e sensorial da cachaça, tais como a qualidade da matéria-prima, as condições de fermentação e os processos de destilação e envelhecimento. Os microorganismos utilizados para o processo fermentativo têm predominância de leveduras, especialmente a Saccharomyces cerevisiae. Ainda, o processo fermentativo é realizado em reciclo sucessivo do fermento. A ocorrência de diferentes espécies e linhagens de leveduras pode interferir negativamente na qualidade do produto final (ALCARDE et al., 2012).

Os diferentes tipos de cachaça variam tanto devido à informalidade, questão cultural, conflitos internos, tributos, concorrência (produtor/produto), quanto 
para a região em que se encontra levando em consideração textura, sabor da bebida e tradição ao longo de sua produção (SCARTON, 2011).

A produção de cachaça artesanal ou de alambique está concentrada nos Estados de Minas Gerais, Rio de Janeiro, Bahia e São Paulo, sendo que os estados mineiro e fluminense contribuem com quase $50 \%$ de toda a produção de cachaça de alambique do Brasil (SAKAI, 2011).

Enquanto a cachaça artesanal é produzida em pequena escala por pequenos produtores, em sua maioria utilizando mão de obra familiar, a cachaça industrial é fabricada em grande escala com maquinários e equipamentos adequados para o mesmo. Estima-se que existam por volta de $40 \mathrm{mil}$ produtores de cachaça artesanal no Brasil. No processo artesanal, a destilação é feita em alambiques de cobre e a fermentação ocorre de forma natural (SAKAI, 2011).

A cachaça industrial é também chamada de aguardente de cana, tem uma produção de grande escala e classificada como industrial devido sua forma de obtenção, que passa pelo processo de fermentação por apenas seis horas, o tempo menor em relação aos outros tipos de cachaça se dá pela utilização de catalisadores químicos (FRANÇA JÚNIOR, 2008).

O metanol é um álcool indesejável quando presente na cachaça e tem origem a partir da degradação da pectina. A molécula de pectina, um polissacarídeo presente na cana-de-açúcar é um composto formado pela associação de centenas de moléculas de ácido galacturônico, que possuem fragmentos de moléculas de metanol, as quais são liberadas durante o processo de fermentação na fabricação do álcool (SILVA; PORTELA; ARAÚJO, 2007).

O metanol tem como fórmula geral $\mathrm{CH}_{3}-\mathrm{OH}$, álcool de estrutura química simples (Figura 1), que à temperatura ambiente apresenta-se líquido, incolor, insípido e com leve odor alcoólico, e seus vapores são tóxicos. É um constituinte naturalmente que podem estar presente nas bebidas alcoólicas, como produto secundário do processo de fermentação (BADOLATO; DURAN, 2000). 


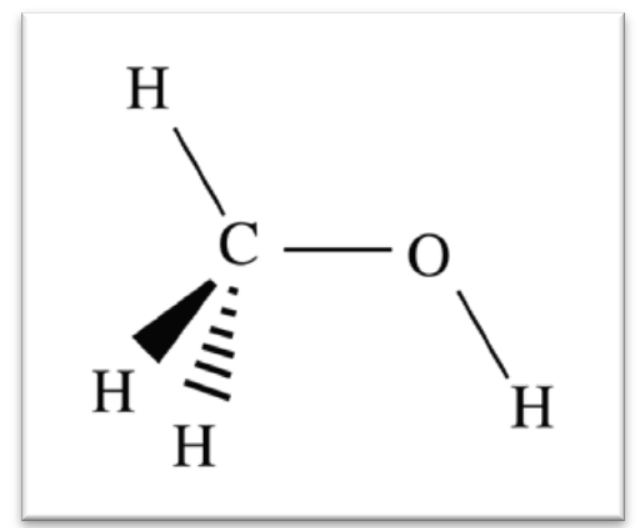

Figura 01. Molécula de metanol.

Fonte: (PATEL, 2010).

No organismo, o metanol é oxidado a ácido fórmico e posteriormente a dióxido de carbono $\left(\mathrm{CO}_{2}\right)$, provocando uma acidose grave (diminuição do $\mathrm{pH}$ sanguíneo), afetando o sistema respiratório. Sua ingestão, mesmo em quantidades reduzidas, em longos períodos de consumo, pode ocasionar cegueira e a morte (SILVA; PORTELA; ARAÚJO, 2007).
A substituição de etanol por metanol, sendo em bebidas alcóolicas e/ou combustíveis são consideradas adulteração devido ao grande risco de intoxicação e com isso esse tipo de substituição é proibido (MARTINS, 2012). Na figura 2 estão descritas as diferenças entre as propriedades físicoquímicas do metanol e etanol.

\begin{tabular}{c|c|c}
\hline & Metanol & Etanol \\
\hline $\begin{array}{c}\text { Fórmula } \\
\text { Molecular }\end{array}$ & $\mathrm{CH}_{3} \mathrm{OH}$ & $\mathrm{C}_{2} \mathrm{H}_{5} \mathrm{OH}$ \\
\hline $\begin{array}{c}\text { Temperatura } \\
\left.\text { de fusăo( }{ }^{\circ} \mathrm{C}\right)\end{array}$ & -98 & -114 \\
\hline $\begin{array}{c}\text { Temperatura } \\
\text { de ebulição } \\
\left({ }^{\circ} \mathrm{C}\right)\end{array}$ & 64 & 78 \\
\hline $\begin{array}{c}\text { Densidade } \\
(\mathrm{g} / \mathrm{mL})\end{array}$ & 0,791 & 0,780 \\
\hline $\begin{array}{c}\text { Indice de } \\
\text { refração } \\
\left(n^{20}{ }_{\mathrm{D}}\right)\end{array}$ & $1,328-1,330$ & $1,360-1363$ \\
\hline
\end{tabular}

Figura 02. Propriedades físico-químicas do metanol e etanol. Fonte: MARTINS, (2012, p. 24).

Deste modo, o presente trabalho teve por objetivo identificar e quantificar o metanol em cachaças brasileiras de diferentes origens, por meio de cromatografia gasosa utilizando a técnica de headspace e comparar os resultados com a legislação brasileira.

\section{METODOLOGIA}

Para conhecimento real da qualidade das diferentes cachaças brasileiras foram utilizadas quatro amostras de cachaças do 
tipo artesanal (alambique) - $(1,2,3,4)$ e quatro amostras de cachaças do tipo industrial $(5,6,7,8)$, todas foram analisadas em duplicata.

As quatro amostras de cachaça artesanal foram adquiridas através de doação, sendo cada uma de alambiques de regiões diferentes, tais como Bahia, Rio Grande do Sul, Mato Grosso do Sul e Goiás. E as quatro amostras de cachaça Industrial foram adquiridas no comércio, sendo as quatro de marcas diferentes.

As análises foram realizadas pelo método de cromatografia gasosa, com detector de ionização de chama (FID) e por headspace, coluna empacotada Carbowax -
20 Molares a $225^{\circ} \mathrm{C}$, para identificação de metanol, nas amostras.

A técnica headspace trata-se de um procedimento de fácil execução. Em um frasco (Vial) de $20 \mathrm{~mL}$ foi adicionado um volume de $1 \mathrm{~mL}$ de $\mathrm{n}$-propanol, utilizado como padrão interno de concentração de $1,6 \mathrm{~g} / \mathrm{L}$ e $1 \mathrm{~mL}$ de amostra (Figura 3), em seguida foi lacrado e colocado no autoamostrador, onde foi aquecida a amostra à temperatura de $80^{\circ} \mathrm{C}$ durante 5 minutos, em seguida um volume de $200 \mu \mathrm{L}$ de amostra foi injetado automaticamente no cromatógrafo em fase gasosa.

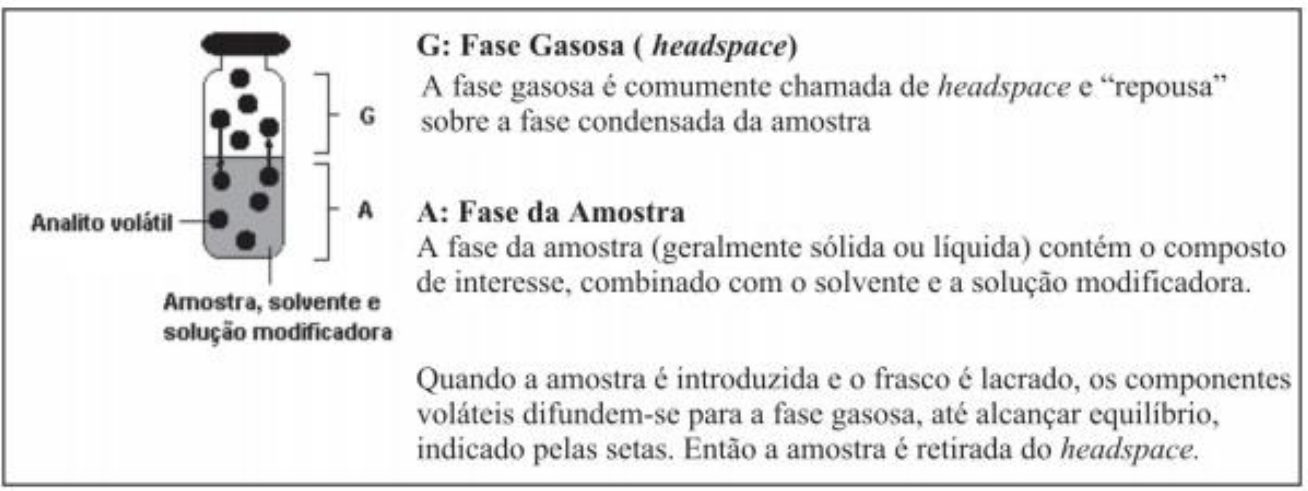

Figura 03. Fases do frasco do headspace.

Fonte: (RESTEK, 2000)

\section{A cromatografia gasosa tem a} vantagem de transferir ao detector $\mathrm{o}$ analito já na forma gasosa, evitando assim os problemas de perdas inerentes à nebulização da amostra, aumentando, consequentemente, a sensibilidade do método.
Para determinar o tempo de retenção do metanol foi realizada uma sequência de diluição seriada 1:10 até 1:10.000. Em seguida foi colocado no autoamostrador e injetado no Cromatógrafo à gás. 


\section{RESULTADOS}

A figura 4 mostra o cromatograma do padrão de metanol, contendo um pico ao qual foi determinado no tempo de retenção de 1,190 minutos.

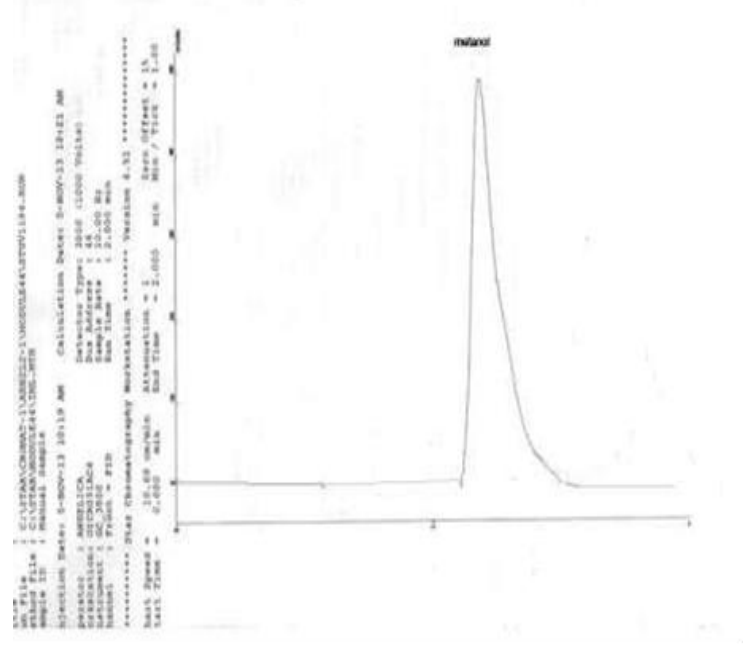

Figura 04. Cromatograma contendo padrão de metanol Fonte: Dados experimentais.

As amostras utilizadas neste estudo, sendo as cachaças artesanais $(1,2,3,4)$ e as industriais $(5,6,7,8)$ não apresentaram teor de metanol.
Foi pré-determinado o tempo de retenção padrão de cada álcool presente na cachaça, sendo eles, metanol, etanol e o npropanol (Tabela 1).

Tabela 01. Tempo de retenção padrão dos álcoois presentes em cachaças.

$\begin{array}{cc}\text { Álcool } & \text { Tempo de retenção (min) } \\ \text { Metanol } & 1,190 \\ \text { Etanol } & 1,276 \\ \text { n-Propanol } & 1,850\end{array}$

Fonte: Dados experimentais.

As amostras de 1 a 8 apresentaram tempo de retenção aproximadamente de 1,276 min e 1,850 min, confirmando então a presença de Etanol e n-Propanol conforme a tabela 2. 
Tabela 02. Tempos de retenção dos álcoois presentes nas amostras de cachaça, determinados por cromatografia em fase gasosa.

\section{Tempo de retenção $(\mathrm{min})$}

$\begin{array}{ccc}\text { Amostra } & \text { Etanol } & \text { n-Propanol } \\ \mathbf{1} & 1,276 & 1,849 \\ \mathbf{2} & 1,275 & 1,850 \\ \mathbf{3} & 1,276 & 1,851 \\ \mathbf{4} & 1,278 & 1,854 \\ \mathbf{5} & 1,280 & 1,857 \\ \mathbf{6} & 1,280 & 1,857 \\ \mathbf{7} & 1,279 & 1,855 \\ \mathbf{8} & 1,279 & 1,856\end{array}$

Fonte: Dados experimentais

\section{DISCUSSÃO}

A cachaça isenta de metanol, ou em valores dentro daqueles considerados seguros $(20 \mathrm{mg} / 100 \mathrm{~mL}$ de etanol anidro) pode ser comercializada, desde que outros contaminantes estejam dentro dos parâmetros aceitos pela legislação (PARAZZI, 2008).

Junior e colaboradores (2006), em pesquisa realizada em cachaças produzidas na região Noroeste do Estado do Rio Grande do Sul, encontraram baixos teores de metanol nas cachaças analisadas.

Miranda e colaboradores (2008), em pesquisa realizada em cachaça durante 0 envelhecimento, na determinação de seu perfil físico químico, encontraram teores de metanol de $6 \mathrm{mg} / 100 \mathrm{~mL}$ de etanol anidro. A ausência ou pequena quantidade de metanol encontrado nas cachaças está explicada, pelo fato da cana de açúcar conter pequena quantidade de pectinas, que quando são hidrolisadas originam o metanol, em destilados.

Souza, Depieri e Clerici (2012) em pesquisa realizada encontraram quantidades de metanol abaixo dos valores estipulados pela legislação. Relatam que o metanol na cachaça pode estar ligado à presença de bagacilhos durante o processo fermentativo, que podem ser degradados a metanol, devido à filtragem ineficiente. Zacaroni e colaboradores (2011) após caracterizar e quantificar contaminantes em aguardentes de cana de açúcar não detectaram o metanol.

\section{CONCLUSÃO}

Durante o processo de fermentação para a produção da cachaça é possível minimizar a produção de metanol, observando o processo de filtração e a matéria prima utilizada, para que não haja a 
presença de matérias pécticas, principais na origem do metanol. Outra forma de encontrar metanol nas cachaças ocorre nas adulterações propositais, ou falsificações das bebidas, o que torna o produto perigoso para o consumo, visto que podem desencadear intoxicações, em alguns casos irreversíveis. 0 presente trabalho tornou-se apenas qualitativo devido a não identificação do metanol.

Portanto, todas as cachaças analisadas não apresenta risco de intoxicação por metanol, de acordo com a legislação brasileira que permite $20 \mathrm{mg} .100 \mathrm{~mL}-1$. Podem ser consumidas com moderação, pois não descarta-se outros riscos, tais como, tontura, vômito, dor de cabeça e até mesmo overdose dependendo da dosagem ingerida.

\section{REFERÊNCIAS}

ALCARDE, A. R. et al. Composição química de aguardentes de cana-de-açúcar fermentadas por diferentes cepas de levedura Saccharomyces cerevisiae. Quim. Nova, v.35, n.8, p.1612-1618, 2012. https://doi.org/10.1590/S010040422012000800022

BADOLATO, E. S. G.; DURAN, M. C. Risco de intoxicação por metanol pela ingestão de bebidas alcoólicas. Arch. Clin. Psychiatry (São Paulo, Impr.), v. 27, n. 2, p. 90-2, 2000.

FRANÇA JÚNIOR, A. Influência do fracionamento no destilado para a otimização da produção da cachaça de alambique: uma prática pedagógica no processo produtivo. 2008. 106f. Dissertação (Mestrado) - Instituto de Agronomia.
Universidade Federal Rural do Rio de Janeiro, Seropédica, 2008.

GAMBINI, R. Espelho índio: a formação da alma brasileira. São Paulo: Terceiro Nome, 2000. p. 96.

JUNIOR, S. B. et al. Composição química da cachaça produzida na região noroeste do Rio Grande do Sul, Brasil. Ciênc. Tecnol. Aliment., v. 26, n. 4, p. 793-798, 2006. https://doi.org/10.1590/S0101$\underline{20612006000400013}$

MAIA, A. B. Componentes secundários da aguardente. Stab, Piracicaba, v. 12, n. 6, p. 29-34, 1994.

MARTINS, G. B. C. Desenvolvimento de uma metodologia portátil para análise de metanol em etanol e detecção de aldeídos visando a utilização em combustíveis e outros produtos comerciais. 2012. Disponível em:

<http://repositorio.bce.unb.br/bitstream/10 482/10324/3/2012_GuilhermeBandeiraCandi doMartins_Parcial.pdf $>$. Acesso em: 22 jul. 2016.

MAZARO, R. A. A cachaça no Brasil e a hospitalidade. In: SIMPÓSIO DE PESQUISA EM COMUNICAÇÃO DA REGIÃO SUDESTE (SIPEC). Anais... v. 10, 2004.

MIRANDA, M. B. et al. Perfil físico-químico de aguardente durante envelhecimento em tonéis de carvalho. Ciência e Tecnologia de Alimentos, v. 28, n. 1, p. 84-89, 2008. https://doi.org/10.1590/S0101$\underline{20612008000500014}$

PARAZZI, C. et al. Avaliação e caracterização dos principais compostos químicos da aguardente de cana-de-açúcar envelhecida em tonéis de carvalho (Quercus sp.). Ciência e Tecnologia de Alimentos, v. 28, n. 1, p. 193-199, 2008. https://doi.org/10.1590/S010120612008000100028 
PATEL, J. Question: Why was methanol used over any other alcohol to carry out the Ugi reaction? 2010. Disponível em: <http://showme.physics.drexel.edu/onsc/arc hives/usefulchem2010-04-27/EXP014-

\%20JanuPatel.html>. Acesso em: 24 jul. 2016.

RESTEK, A. Technical guide for static headspace analysis using GC. Restek Corp, 2000. p.11-12.

SAKAI, R. H. Cachaça. 2011. Disponível em: <http://www.agencia.cnptia.embrapa.br/ges tor/cana-de-

acucar/arvore/CONT000fiog1ob502wyiv80z4 s473agi63ul.html>. Acesso em: 24 jul. 2016.

SCARTON, L. M. Governança na cadeia da cachaça artesanal: o caso do grupo Alambiques Gaúchos. 2011. Disponível em: <http://www.lume.ufrgs.br/bitstream/handl e/10183/30198/000780088.pdf?sequence=1 > Acesso em: 24 jul. 2016.

SILVA, L.M.F.; PORTELA, A. L. O; ARAÙJO, A. $D$. A determinação do teor de álcool metílico (metanol) em amostras de cachaças vendidas na região Norte do Ceará [on line]. ABQ-RN, $2007 . \quad$ Disponível em: <http://www.abq.org.br/cbq/2007/trabalhos /4/4-628-696.htm>. Acesso em 23 jul. 2016.

SOUZA, R. A.; DEPIERE, M.; CLERICI, M. T. P. $\mathrm{S}$. Perfil físico-químico de cachaças industriais produzidas na região sudeste do Brasil na safra 2008/2009. Revista Agrotecnologia, v.3, n.1, p.91-110, 2012. https://doi.org/10.12971/2179-5959

ZACARONI, L. M. et al. Caracterização e quantificação de contaminantes em aguardentes de cana. Quim. Nova, v. 34, n. 2, p.320-324, 2011. https://doi.org/10.1590/S0100$\underline{40422011000200026}$ 